\title{
Optical design of the Origins space telescope
}

James A. Corsetti ${ }^{1}$, Edward G. Amatucci ${ }^{1}$, Ruth C. Carter ${ }^{1}$, Tom D’Asto ${ }^{2}$, Michael J. DiPirro'1, Benjamin Gavares ${ }^{3}$, Joseph M. Howard ${ }^{1}$, David T. Leisawitz ${ }^{1}$, Gregory E. Martins ${ }^{1}$, Margaret Meixner ${ }^{4}$, and Len Seals ${ }^{1}$

${ }^{1}$ NASA GSFC, 8800 Greenbelt Rd, Greenbelt, MD 20771

${ }^{3}$ Northrup Grumman, 5010 Herzel PI, Beltsville, MD 20705

${ }^{2}$ ATA Aerospace, 7474 Greenway Center Dr, Suite 500, Greenbelt, MD 20770 ${ }^{4}$ STScl, 3700 San Martin Dr, Baltimore, MD 21218

\section{Introduction}

Origins is one of four large missions currently under study for the 2020 Decadal Survey in Astronomy and Astrophysics. Sensitive in the mid- and far-infrared spectrum (between 2.8 and $588 \mu \mathrm{m})$, Origins main science themes are:

1) How do galaxies form stars, build up metals, and grow thei supermassive black holes from reionization to today?

2) How do the conditions for habitability develop during the process of planet formation?

3) Do planets orbiting M-dwarf stars support life?

\section{Telescope Overview}

Origins is a three-mirror anastigmat (TMA) like the Jame Webb Space Telescope (JWST), that includes three powered optics (the primary, secondary, and tertiary mirrors) as well as a flat field-steering mirror (FSM). The telescope image surface is concave, with its center of curvature located at the FSM surface which is also the location of the telescope's exit pupil. By constraining the design in this way, defocus does not result when scanning the FSM.

The TMA architecture enables the required wavefron performance (diffraction-limited at $\lambda=30 \mu \mathrm{m}$ ) over a relatively large field of view. Origins is designed to fit within its launch vehicle without requiring deployments (including the primary and secondary) which helps to reduce telescope complexity and cost. The primary size (5.9 m diameter) and on-axis configuration are based on this requirement, maximizing collecting area while meeting packaging constraints. Origins utilizes 18 'keystone' shaped segments arranged in two annuli to form its primary mirror. Such a segmentation scheme is desirable over hexagons as it maximizes the collecting area of the mirror in a given fairing size while also reducing the effects of diffraction. Only two optical prescriptions are required for the segments (those of the inner and outer annuli). Because of its high stiffness, low density, low CTE, and high therma conductivity, $\mathrm{Be} \mathrm{O}-30$ is baselined for the optical components and instrument structure.
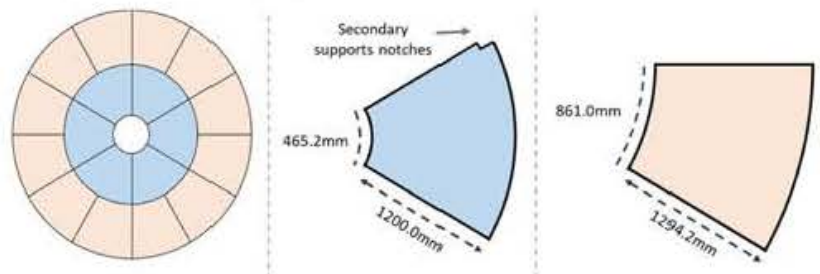
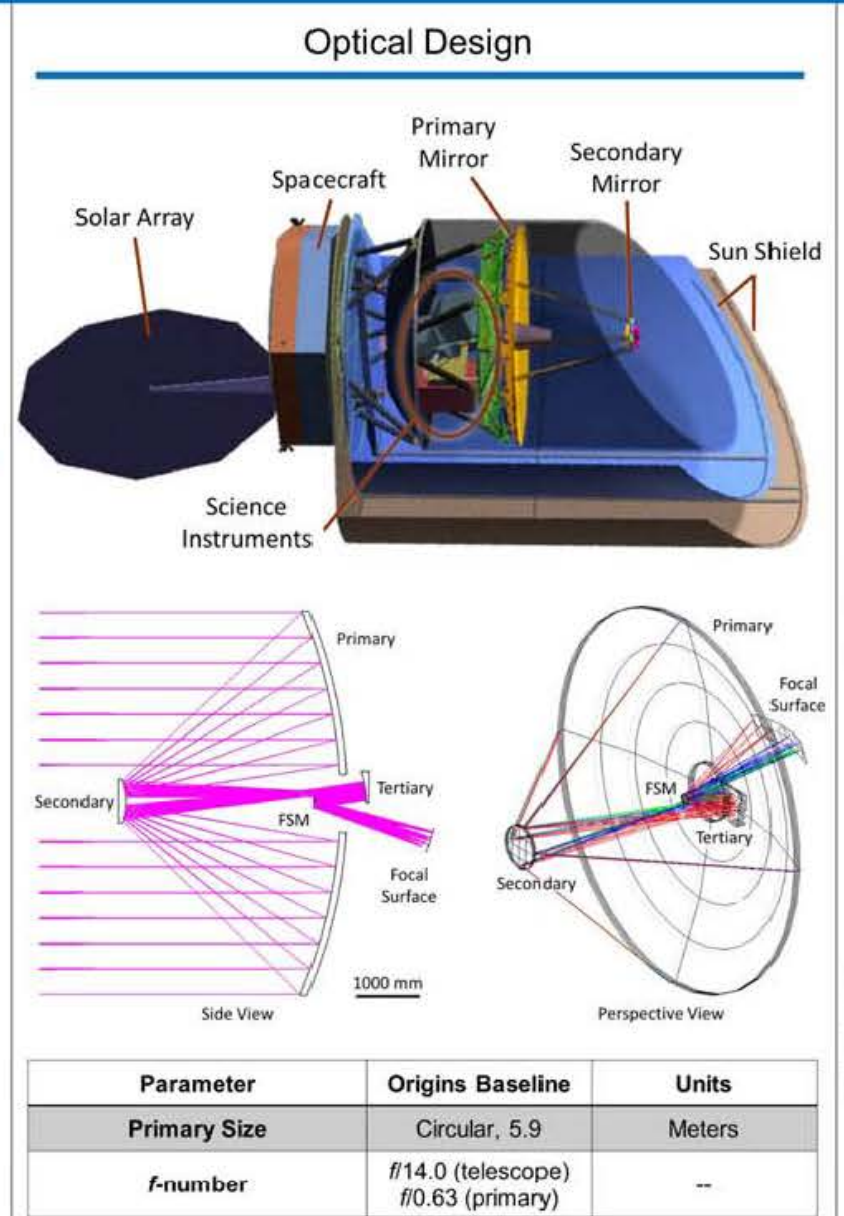

\begin{tabular}{|c|c|c|}
\hline Effective Focal Length & $f / 0.63$ (primary) & \\
\hline Field of View & 82.6 & Meters \\
\hline Waveband & $46 \times 15$ & Arcmin \\
\hline Operating Temp. & $2.8-588$ & Microns \\
\hline Optical Performance & 4.5 & Kelvin \\
\hline Design Form & Diffraction limited at $\lambda=30 \mu \mathrm{m}$ \\
\hline Mirror Coatings & $\begin{array}{c}\text { Three Mirror Anastigmat } \\
\text { Obstructed (on-axis pupil) }\end{array}$ \\
\hline $\begin{array}{c}\text { Optical and Structural } \\
\text { Material }\end{array}$ & Protected Gold \\
\hline
\end{tabular}

\section{Science Instruments}

The baseline Origins design includes three instruments

1) Origins Survey Spectrometer (OSS) with Fourier Transform Spectrometer (FTS) and etalon

2) Mid-infrared Spectrometer Camera Transit Spectrometer (MISC-T)

3) Far-Infrared Imager Polarimeter (FIP)

Upscope options for Origins include a fourth instrument, Heterodyne Receiver for Origins (HERO), imaging camera module (MISC I), and increased fields of view for the OSS and FIP instruments.
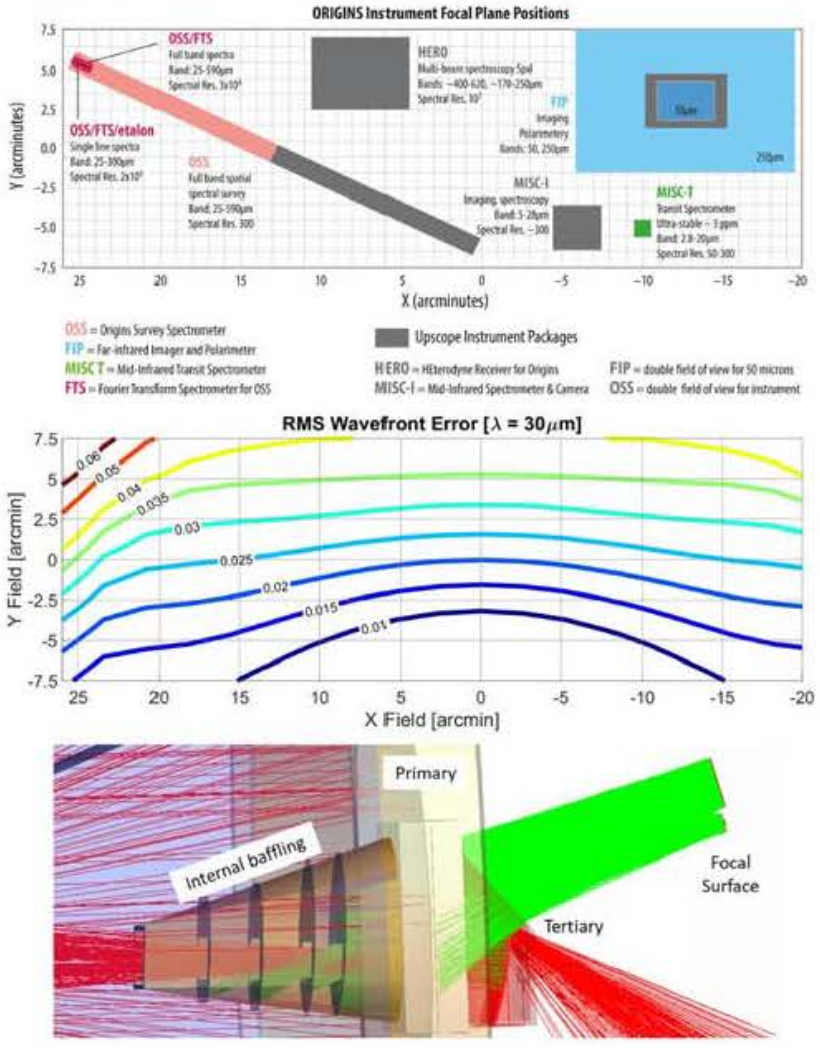

REFERENCES:

1. Origins Final Report. NASA GSFC. August 2019 